\title{
SYNTHESIS AND STRUCTURE OF OSMIUM COMPLEX $\left[\mathrm{Ph}_{4} \mathrm{P}_{2}\left[\mathrm{OsBr}_{6}\right]\right.$
}

\author{
V.V. Sharutin, vvsharutin@rambler.ru \\ O.K. Sharutina, sharutinao@mail.ru \\ V.S. Senchurin, senvl@rambler.ru \\ South Ural State University, Chelyabinsk, Russian Federation
}

\begin{abstract}
Tetraphenylphosphonium hexabromoosmate, $\left[\mathrm{Ph}_{4} \mathrm{P}\right]_{2}\left[\mathrm{OsBr}_{6}\right]$ (I), has been structurally characterized after synthesis by interaction of sodium hexabromoosmate with tetraphenylphosphonium bromide in dimethyl sulfoxide. The phosphorus atoms of $\left[\mathrm{Ph}_{4} \mathrm{P}\right]^{+}$cations have distorted tetrahedral coordination geometry (CPC angles are $106.23(11)^{\circ}-113.23(10)^{\circ}$ ), the $\mathrm{P}-\mathrm{C}$ bond lengths are 1.791(2)-1.801(2) $\AA$ ). In octahedral $\left[\mathrm{OsBr}_{6}\right]^{2-}$ anions the $\mathrm{Os}-\mathrm{Br}$ bond lengths equal 2.4752(2)-2.5020(3) $\AA$, trans-BrOsBr angles are $180^{\circ}$.

Keywords: sodium hexabromoosmate, tetraphenylphosphonium bromide, dimethyl sulfoxide, complex $\left[\mathrm{Ph}_{4} \mathrm{P}\right]_{2}\left[\mathrm{OsBr}_{6}\right]$, crystal structure, $\mathrm{X}$-ray diffraction analysis.
\end{abstract}

\section{Introduction}

Complexes containing $\left[\mathrm{OsBr}_{6}\right]^{2-}$ anions are some of the least structurally characterized ionic osmium complexes [1]; among them there is only one complex with phosphonium cations, $\left[\mathrm{Ph}_{3} \mathrm{PH}\right]_{2}^{+}\left[\mathrm{OsBr}_{6}\right]^{2-}[2]$.

In the present paper the synthesis and structure of osmium complex $\left[\mathrm{Ph}_{4} \mathrm{P}^{+}{ }_{2}\left[\mathrm{OsBr}_{6}\right]^{2-}(\mathbf{1})\right.$ has been described.

\section{Experimental}

Synthesis of $\left[\mathbf{P h}_{4} \mathbf{P}\right]_{2}^{+}{ }_{2}\left[\mathbf{O s B r}_{6}\right]^{2-}(\mathbf{1})$. A mixture of $0.035 \mathrm{~g}(0.08 \mathrm{mmol})$ of tetraphenylphosphonium bromide and $0.030 \mathrm{~g}(0.04 \mathrm{mmol})$ of sodium hexabromoosmate was dissolved by stirring in $2 \mathrm{~mL}$ of dimethyl sulfoxide. After evaporation of the solvent, formation of dark brown crystals was observed; they were filtered off and dried. $0.046 \mathrm{~g}(82 \%)$ of the complex was obtained, m.p. $320^{\circ} \mathrm{C}$. IR $\left(v, \mathrm{~cm}^{-1}\right): 3053$, 1583, 1482, 1437, 1313, 1186, 1107, 1025, 995, 761, 752, 721, 690, 663, 531, 522. Found, \%: C 42.49, H 3.06. Anal. calc. for $\mathrm{C}_{48} \mathrm{H}_{40} \mathrm{P}_{2} \mathrm{Br}_{6} \mathrm{Os}(\mathrm{M}=1348.37)$, \%: C 42.74, $\mathrm{H} 2.97$.

IR-spectrum was recorded on a Bruker Tensor $27 \mathrm{IR}$ spectrometer in $\mathrm{KBr}$ pellet.

The X-ray diffraction experiment for crystal 1 was carried out on a Bruker D8 Quest diffractometer (Mo $\mathrm{K}_{\alpha}$ radiation, $\lambda=0.71073 \AA$, graphite monochromator). Data collection, their editing, and refinement of the unit cell parameters, as well as accounting for absorption, were carried out using the SMART and SAINT-Plus programs [3]. All calculations for structure determination and refinement were carried out using the SHELXL/PC program [4]. Structure 1 was determined by the direct method and refined by the least-squares method in anisotropic approximation for non-hydrogen atoms. The main crystallographic data and structure refinement details are given in Table 1. The main bond lengths and bond angles are listed in Table 2.

The full tables of atomic coordinates, bond lengths, and bond angles were deposited with the Cambridge Crystallographic Data Centre (№ 1000138; deposit@ccdc.cam.ac.uk; http://www.ccdc.cam.ac.uk).

Crystallographic data, the experimental and structure refinement parameters for compound 1

Table 1

\begin{tabular}{|c|c|}
\hline Parameter & Value \\
\hline Formula & $\mathrm{C}_{48} \mathrm{H}_{40} \mathrm{P}_{2} \mathrm{Br}_{6} \mathrm{Os}$ \\
\hline$M$ & 1348.37 \\
\hline$T, K$ & $296(2)$ \\
\hline Crystal system & Triclinic \\
\hline Space group & $\mathrm{P}-1$ \\
\hline
\end{tabular}


Organometallic chemistry

Table 1 (end)

\begin{tabular}{|c|c|}
\hline Parameter & Value \\
\hline$a, \AA$ & $10.2879(4)$ \\
\hline$b, \AA$ & $10.5331(4)$ \\
\hline$c, \AA$ & $12.1824(5)$ \\
\hline$\alpha, \operatorname{deg}$ & $92.7640(10)$ \\
\hline$\beta, \operatorname{deg}$ & $99.9850(10)$ \\
\hline$\gamma, \operatorname{deg}$ & $116.3110(10)$ \\
\hline$V, \AA^{3}$ & $1153.68(8)$ \\
\hline$Z$ & 1 \\
\hline$\rho($ calc. $), \mathrm{g} / \mathrm{cm}^{3}$ & 1.941 \\
\hline$\mu, \mathrm{mm}^{-1}$ & 8.061 \\
\hline$F(000)$ & 644.0 \\
\hline Crystal size, $\mathrm{mm}$ & $0.32 \times 0.26 \times 0.13$ \\
\hline$\theta$ Range of data collection, deg & $5.92-52.86$ \\
\hline Range of refraction indices & $-12 \leq \mathrm{h} \leq 12,-13 \leq \mathrm{k} \leq 13,-15 \leq 1 \leq 15$ \\
\hline Measured reflections & 36441 \\
\hline Independent reflections & $4730\left(\mathrm{R}_{\text {int }}=0.0245\right)$ \\
\hline Refinement variables & 259 \\
\hline GOOF & 1.105 \\
\hline$R$ factors for $\mathrm{F}^{2}>2 \sigma\left(\mathrm{F}^{2}\right)$ & $R_{1}=0.0171, w R_{2}=0.0416$ \\
\hline $\mathrm{R}$ factors for all reflections & $R_{1}=0.0195, w R_{2}=0.0426$ \\
\hline Residual electron density ( $\min / \max ), \mathrm{e} / \mathrm{A}^{3}$ & $0.35 /-0.66$ \\
\hline
\end{tabular}

Table 2

Selected bond lengths (d) and bond angles $(\omega)$ in the structure of compound 1

\begin{tabular}{|c|c|c|c|}
\hline Bond & $\boldsymbol{d}, \boldsymbol{\AA}$ & Angle & $\boldsymbol{\omega}, \mathbf{d e g}$ \\
\hline $\mathrm{Os}(1)-\mathrm{Br}\left(1^{1}\right)$ & $2.4985(2)$ & $\mathrm{Br}\left(1^{1}\right) \mathrm{Os}(1) \mathrm{Br} 1$ & 180.0 \\
\hline $\mathrm{Os}(1)-\mathrm{Br}(1)$ & $2.4986(2)$ & $\mathrm{Br}(2) \mathrm{Os}(1) \mathrm{Br} 1$ & $91.620(8)$ \\
\hline $\mathrm{Os}(1)-\mathrm{Br}(2)$ & $2.4752(2)$ & $\mathrm{Br}\left(2^{1}\right) \mathrm{Os}(1) \mathrm{Br} 1$ & $88.380(8)$ \\
\hline $\mathrm{Os}(1)-\mathrm{Br}(3)$ & $2.5019(3)$ & $\mathrm{C}(1) \mathrm{P}(1) \mathrm{C} 31$ & $106.23(11)$ \\
\hline $\mathrm{Os}(1)-\mathrm{Br}\left(3^{1}\right)$ & $2.5020(3)$ & $\mathrm{C}(1) \mathrm{P}(1) \mathrm{C} 11$ & $109.65(11)$ \\
\hline $\mathrm{P}(1)-\mathrm{C}(1)$ & $1.798(2)$ & $\mathrm{C}(31) \mathrm{P}(1) \mathrm{C} 11$ & $109.57(11)$ \\
\hline $\mathrm{P}(1)-\mathrm{C}(31)$ & $1.800(2)$ & $\mathrm{C}(21) \mathrm{P}(1) \mathrm{C} 1$ & $113.23(10)$ \\
\hline $\mathrm{P}(1)-\mathrm{C}(21)$ & $1.791(2)$ & $\mathrm{C}(21) \mathrm{P}(1) \mathrm{C} 31$ & $108.47(10)$ \\
\hline $\mathrm{P}(1)-\mathrm{C}(11)$ & $1.801(2)$ & $\mathrm{C}(21) \mathrm{P}(1) \mathrm{C} 11$ & $109.60(11)$ \\
\hline Symmetry transformation: ${ }^{1}-\mathrm{x}, 1-\mathrm{y}, 1-\mathrm{z}$ & \\
\hline
\end{tabular}

\section{Results and Discussion}

It is known that the synthesis of osmium complex $\left[\mathrm{Ph}_{3} \mathrm{PH}_{2}^{+}\left[\mathrm{OsBr}_{6}\right]^{2-}\right.$ was carried out by consecutive addition of triphenylphosphine, glacial acetic acid and acetic anhydride to the dichloromethane solution of tetrabutylammonium hexabromoosmate, and heating of the reaction mixture for 3 days at 55-60 ${ }^{\circ} \mathrm{C}$, with the yield equaling $29 \%$ [2].

We have ascertained that the reaction of sodium hexabromoosmate with tetraphenylphosphonium bromide in dimethyl sulfoxide leads to formation of air-stable dark brown crystals of tetraphenylphosphonium hexabromoosmate (1), isolated from the reaction mixture with the yield equaling $82 \%$ :

\section{DMSO}

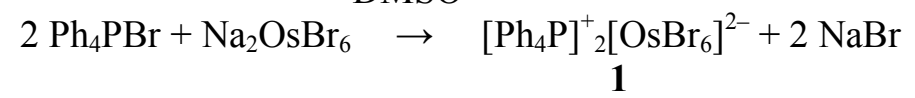


According to the X-ray diffraction data, phosphorus atoms in tetraphenylphosphonium cations have slightly distorted tetrahedral coordination geometry: CPC angles lie within the range 106.23(11) $-113.23(10)^{\circ}, \mathrm{P}-\mathrm{C}$ distances are 1.791(2)-1.801(2) ̊̊) (Fig. 1).

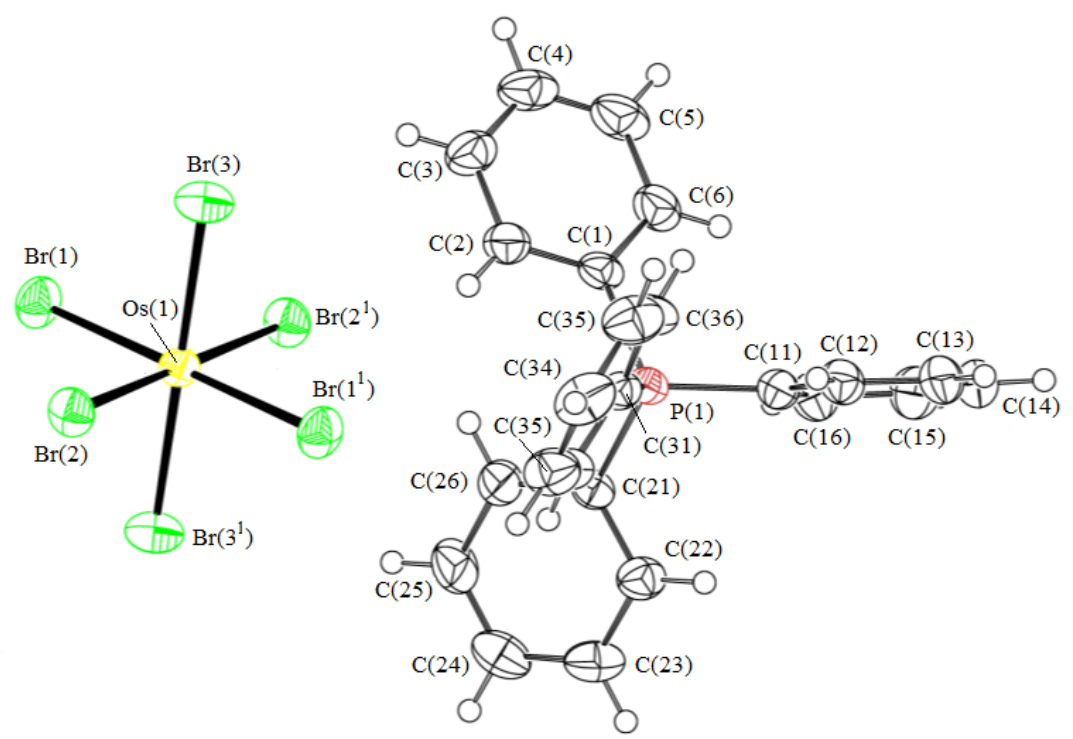

Fig. 1. Structure of complex 1

In centrosymmetric octahedral $\left[\mathrm{OsBr}_{6}\right]^{2-}$ anions, the Os-Br bond lengths equal 2.4752(2)-2.5020(3) $\AA$, trans-BrOsBr angles are $180^{\circ}$. Two tetraphenylphosphonium cations are bonded with hexabromoosmate anion by intermolecular $\mathrm{H} \cdots$ Br hydrogen bonds (2.90 and 3.01 $\AA$ ) (Fig. 2).

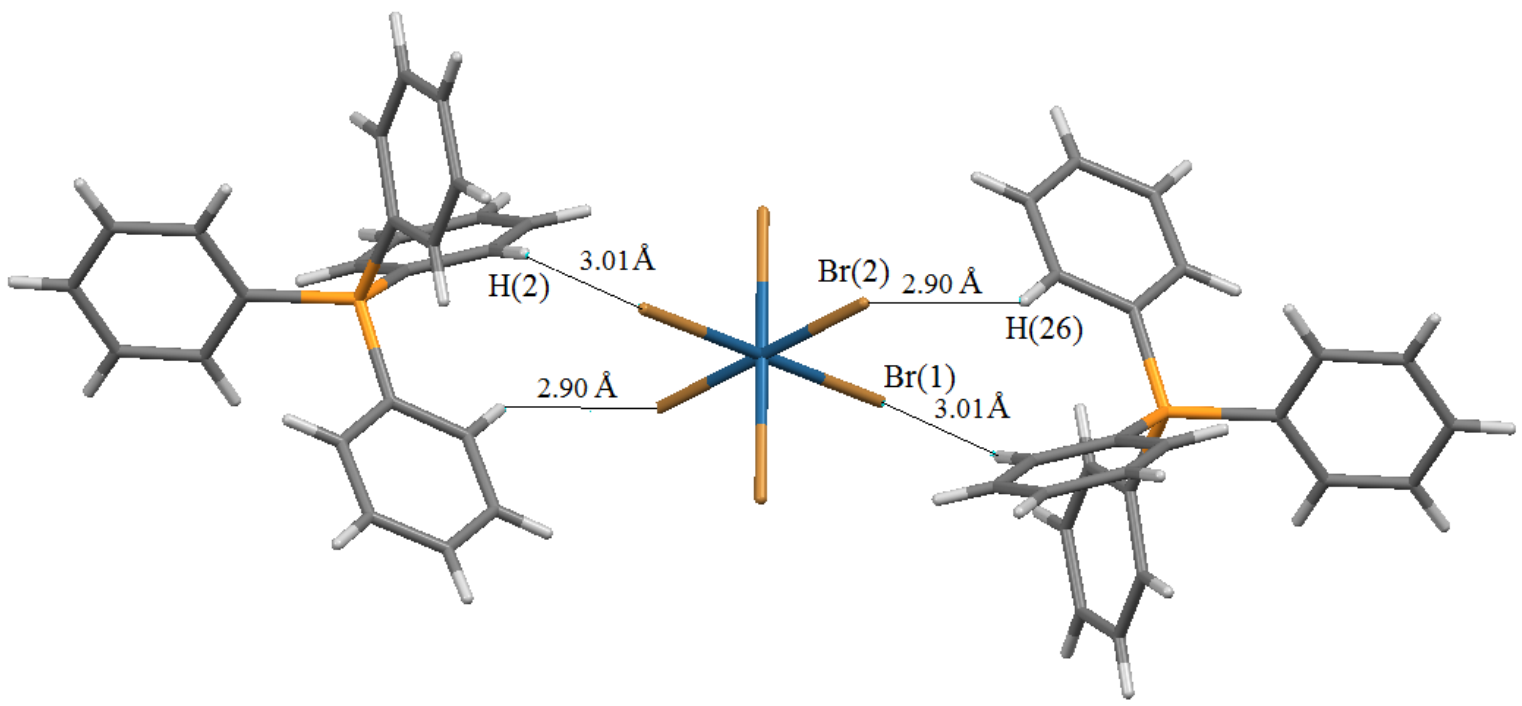

Fig. 2. The system of hydrogen bonds in the crystal of complex 1

\section{Conclusions}

Thus, the osmium complex with the tetrahedral tetraphenylphosphonium cation and the centrosymmetric octahedral Os-, Br-containing $\left[\mathrm{OsBr}_{6}\right]^{2-}$ anion has been synthesized and structurally characterized for the first time. Formation of crystal structure occurs due to intermolecular $\mathrm{H} \cdots \mathrm{Br}$ hydrogen bonds between tetraphenylphosphonium cations and hexabromoosmate anions. 


\title{
References
}

1. Cambridge Crystallografic Database. Release 2015. Cambridge.

2. Robinson P.D., Hinckley C.C., Matusz M., Kibala P.A. Acta Cryst. 1988. Vol. C44. P. 619-621.

3. Bruker (1998). SMART and SAINT-Plus.Versions 5.0.Data Collection and Processing Software for the SMART System.Bruker AXS Inc., Madison, Wisconsin, USA.

4. Bruker (1998). SHELXTL/PC.Versions 5.10. An Integrated System for Solving, Refining and Displaying Crystal Structures From Diffraction Data. Bruker AXS Inc., Madison, Wisconsin, USA.

Received 18 May 2015

Удк У546.97+546.185+547.53.024+548.312.5

DOI: $10.14529 /$ chem 150402

\section{СИНТЕЗ И СТРОЕНИЕ КОМПЛЕКСА ОСМИЯ $\left[\mathrm{Ph}_{4} \mathrm{P}_{2}\left[\mathrm{OsBr}_{6}\right]\right.$}

\author{
В.В. Шарутин, О.К. Шарутина, В.С. Сенчурин \\ Южно-Уральский государственный университет, г. Челябинск
}

\begin{abstract}
Взаимодействием гексабромоосмата натрия с бромидом тетрафенилфосфония в диметилсульфоксиде синтезирован и структурно охарактеризован гексабромоосмат тетрафенилфосфония $\left[\mathrm{Ph}_{4} \mathrm{P}\right]_{2}\left[\mathrm{OsBr}_{6}\right]$ (I). Атомы фосфора в катионах $\left[\mathrm{Ph}_{4} \mathrm{P}\right]^{+}$имеют искаженную тетраэдрическую координацию (углы СРС $106.23(11)^{\circ}-113.23(10)^{\circ}$ ), расстояния Р-C составляют 1.791(2)-1.801(2) А). В октаэдрических анионах $\left[\mathrm{OsBr}_{6}\right]^{2-}$ длины связей Os-Br равны 2.4752(2)-2.5020(3) А, углы транс-BrOsBr составляют $180^{\circ}$.

Ключевые слова: гексабромоосмат натрия, бромид тетрафенилфосфония, диметилсульфоксид, комплекс $\left[\mathrm{Ph}_{4} \mathrm{P}_{2}\left[\mathrm{OsBr}_{6}\right]\right.$, структура, рентгеноструктурный анализ.
\end{abstract}

Шарутин Владимир Викторович - доктор химических наук, профессор, старший научный сотрудник УНИД, Южно-Уральский государственный университет. 454080, г. Челябинск, пр. им. В.И. Ленина, 76. E-mail: vvsharutin@rambler.ru

Шарутина Ольга Константиновна - доктор химических наук, профессор, кафедра аналитической химии, Южно-Уральский государственный университет. 454080, г. Челябинск, пр. им. В.И. Ленина, 76. E-mail: sharutinao@mail.ru

Сенчурин Владислав Станиславович - кандидат химических наук, кафедра органической химии, Южно-Уральский государственный университет. 454080, г. Челябинск, пр. им. В.И. Ленина, 76. E-mail: senvl@rambler.ru

Поступила в редакцию 18 мая 2015 2.

\section{ОБРАЗЕЦ ЦИТИРОВАНИЯ}

Sharutin, V.V. Synthesis and structure of osmium complex $\left[\mathrm{Ph}_{4} \mathrm{P}\right]_{2}\left[\mathrm{OsBr}_{6}\right]$ / V.V. Sharutin, O.K. Sharutina, V.S. Senchurin // Вестник ЮУрГУ. Серия «Химия». 2015. - T. 7, № 4. - C. 13-16. DOI: 10.14529/chem150402

\section{FOR CITATION}

Sharutin V.V., Sharutina O.K., Senchurin V.S. Synthesis and Structure of Osmium Complex $\left[\mathrm{Ph}_{4} \mathrm{P}\right]_{2}\left[\mathrm{OsBr}_{6}\right]$. Bulletin of the South Ural State University. Ser. Chemistry. 2015, vol. 7, no. 4, pp. 13-16. DOI: 10.14529/chem150402 